\title{
Educación y desarrollo sostenible: la creatividad de la naturaleza para innovar en la formación humana
}

\author{
Javier Collado-Ruano
}

Universidad Nacional de Educación

(UNAE) del Ecuador. Ecuador.

javier.collado@unae.edu.ec

orcid.org/0000-0003-0063-6642

\section{Resumen}

El artículo reflexiona sobre los desafíos de la formación humana para cumplir los Objetivos de Desarrollo Sostenible (ODS) propuestos por las Naciones Unidas. La educación y el desarrollo sostenible constituyen el eje temático que guía la configuración epistemológica del estudio, enmarcado en el campo de la filosofía de la educación. En el trabajo se combina la metodología transdisciplinar, el marco teórico de la gran historia y el principio de biomímesis, con el objetivo primordial de reforzar los lazos entre la educación y la sostenibilidad. Como resultado de la discusión, la investigación muestra diferentes estrategias coevolutivas que los ecosistemas desarrollan en la naturaleza para implementarlas en la práctica pedagógica. El desarrollo sostenible implica una formación humana orientada a respetar los límites biofísicos establecidos por los ecosistemas naturales. La conclusión principal apunta la necesidad de adoptar nuevos abordajes biomiméticos y transdisciplinares en la formación humana para solucionar los desafios de insostenibilidad planetaria que tiene la huella ecológica y social en los albores del siglo XXI.

\section{Palabras clave}

Educación; desarrollo sostenible; filosofía de la educación; creatividad; naturaleza; epistemología (Fuente: Tesauro de la Unesco).

Recepción: 10-03-2016 | Envío a pares: 02-05-2016 | Aceptación por pares: 21-10-2016 | Aprobación: 10-11-2016 


\title{
Education and Sustainable Development: The Creativity of Nature for Innovation in Human Education
}

\begin{abstract}
The article reflects on the challenges that exist in terms of human education to comply with the United Nations Sustainable Development Goals (SDS). Education and sustainable development are the thematic pivotal point that guide the epistemological shape of the study, which is framed by the philosophy of education. The work combines the transdisciplinary methodology, the theoretical framework of great history and the biomimesis principle with the primary objective of strengthening the ties between education and sustainability. As a result of the discussion, the research shows different co-evolutionary strategies that ecosystems develop in nature, so as to implement them in teaching practice. Sustainable development implies human training aimed at respecting the biophysical limits established by natural ecosystems. The main conclusion points to the need to adopt new biomimetic and transdisciplinary approaches in human education to solve the challenges of planetary unsustainability caused by the ecological and social footprint of man at the dawn of the 21st century.
\end{abstract}

\section{Keywords}

Education; sustainable development; philosophy of education; creativity; nature; epistemology (Source: Unesco Thesaurus). 


\section{Educação e desenvolvimento sustentável: a criatividade da natureza para inovar na formação humana}

\section{Resumo}

O artigo reflete sobre os desafios da formação humana para cumprir os Objetivos de Desenvolvimento Sustentável propostos pelas Nações Unidas. A educação e o desenvolvimento sustentável constituem o eixo temático que guia a configuração epistemológica do estudo, enquadrado no campo da filosofia da educação. No trabalho, combinam-se a metodologia transdisciplinar, o marco teórico da grande história e o princípio de biomimética, com o objetivo primordial de reforçar os laços entre a educação e a sustentabilidade. Como resultado da discussão, a pesquisa mostra diferentes estratégias coevolutivas que os ecossistemas desenvolvem na natureza para implementá-las na prática pedagógica. O desenvolvimento sustentável implica em uma formação humana orientada para respeitar os limites biofísicos estabelecidos pelos ecossistemas naturais. A conclusão principal aponta a necessidade de adotar novas abordagens biomiméticas e transdisciplinares na formação humana para solucionar os desafios da falta de sustentabilidade no planeta, que tem a marca ecológica e social no início do século XXI.

\section{Palavras-chave}

Educação; desenvolvimento sustentável; filosofia da educação; criatividade; natureza; epistemologia (Fonte: Tesauro da Unesco). 


\section{Introducción}

Hablar sobre la naturaleza de la creatividad es hablar sobre la capacidad que determinados seres vivos tienen para crear, adaptar y modelar nuevos ambientes. Es una capacidad intrínseca al propio fenómeno de la vida. La vida es un continuum que surgió en la Tierra hace unos 3800 millones de años, después de una larga evolución del universo que todavía está en permanente reestructuración energética. Entonces, estudiar la naturaleza de la creatividad significa estudiar la creatividad de la naturaleza, la creatividad de la vida y la creatividad de los fenómenos cósmicos. ¿Pero cuál es la fuente de toda esa creatividad? ¿Cuál es el papel de la creatividad en el desarrollo humano sostenible de su caminar histórico? ¿Cómo podemos comprender la creatividad infinita que está inscrita en el ADN de los procesos coevolutivos de la naturaleza del cosmos? ¿Cuál es el papel de la educación en el siglo XXI para potenciar la creatividad en la formación humana? Con esas cuestiones de fondo, el presente ensayo reflexiona rigurosamente sobre la naturaleza de la creatividad en las estrategias y los procesos coevolutivos de la vida en la naturaleza y el cosmos que tiene como objetivo principal la identificación de sus principios operacionales para copiarlos y aplicarlos biomiméticamente en los sistemas socioeconómicos humanos, con el propósito de crear un horizonte civilizatorio más sostenible y resiliente, conforme es demandado urgentemente por los Objetivos de Desarrollo Sostenible (ODS) de las Naciones Unidas para 2030. En ese abordaje transdisciplinar, biomimético y complejo emerge una nueva perspectiva de educación sostenible para la formación humana del siglo XXI que se explica en las siguientes páginas.

\section{La metodología transdisciplinar: crear lazos creativos entre la educación y la sostenibilidad}

Un breve sumario de la coevolución ecosistémica en Gaia' es suficiente para comprender la

$1 \quad$ Gaia es la diosa primigenia que personifica la Tierra en la mitología griega. infinita creatividad de la vida en la naturaleza. Su amplia complejidad necesita herramientas epistémicas que tengan en cuenta los diferentes niveles en los que operan las distintas leyes biofísicas. La idea de interconexión entre los seres humanos y las demás formas de vida nos lleva a revisar el concepto de bioética a través del estudio transdisciplinar de los procesos coevolutivos que la vida viene desarrollando desde su aparición en la Tierra. El "milagro cósmico de la vida" es un desafío transdisciplinar que la bioética debe integrar para salvaguardar la gran biodiversidad que coevoluciona en Gaia (Collado, 2016a). Por este motivo, el presente artículo combina el marco teórico de la gran historia acuñado y fundamentado teóricamente por los historiadores David Christian (2010) y Fred Spier (2011), con la metodología transdisciplinar propuesta por el físico nuclear Basarab Nicolescu (2014): niveles de realidad, lógica del tercero incluido y complejidad. Esta simbiosis teórica y metodológica supone un abordaje epistemológico que comprende al ser humano como una parte integrante de la totalidad cósmica autopiética, y alberga, además, el imperativo ético de desarrollar una cultura de paz para el cumplimiento de los ODS. Para alcanzar los ODS en 2030, la educación formal, no formal e informal representa una herramienta esencial para transformar creativamente la sociedad-mundo.

De acuerdo con la obra Los siete saberes necesarios para la educación del futuro, escrita por el sociólogo Edgar Morin en 1999 bajo la invitación del director del proyecto transdisciplinar Educación para un futuro sostenible de la Organización de las Naciones Unidas para la Educación, la Ciencia y la Cultura (Unesco), "enseñar la condición humana significa enseñar la condición cósmica, física y terrestre del individuo-sociedad-especie" (pp. 21-23). Desde esos horizontes intelectuales visionarios, toda educación que pretenda ser universal tiene que tener en cuenta los diferentes niveles de realidad gnoseológicos y ontológicos que constituyen la identidad multidimensional del individuo-sociedad-especie: como 
individuo de una comunidad local específica, como ciudadano o ciudadana de una sociedad determinada perteneciente a una comunidad o Estado nación y como una misma especie cosmobiogenética en constante proceso de coevolución con el medio ambiente. Una triidentidad humana abierta a la diversidad cultural infinita de la ciudadanía planetaria en su propia unidad como especie. Del mismo modo que la propia ontología estructura la naturaleza en diferentes niveles de realidad, el ser humano tiene diferentes estratos, niveles y planos de percepción gnoseológica que estructuran y concretizan su complejidad histórica en su contexto cosmológico, de ahí que también se pueda adicionar la vertiente identidaria del ciber-espacio-tiempo: la identidad virtual.

Esta visión epistemológica moriana se encuentra en armonía con la educación cósmica formulada en 1935 por la bióloga, médica, psiquiatra, antropóloga, filósofa, educadora y pedagoga María Montessori, que diseña un abordaje pedagógico para modelar la formación humana a través de la complejidad adyacente en los niveles de identidad que componen al género humano, sin caer en lógicas reduccionistas, unidimensionalizantes u homogeneizadoras. Nuestra identidad está construida a partir de múltiples dependencias. Es una construcción original a partir de múltiples relaciones. Toda cultura es más o menos híbrida, mestiza, hecha de cruces, retroalimentaciones... No existen culturas acabadas ni perfectas. Toda cultura lleva en sí misma suficiencias, insuficiencias, funcionalidades, disfuncionalidades... "El bucle conceptual eco-bio-antropo-social es un bucle en el que el pensamiento de la complejidad natural debe permitir desarrollar el pensamiento de la complejidad social y política" (Morin, 1983, p. 120). En esa línea de pensamiento, el método Montessori es un conjunto de saberes, prácticas y propuestas caracterizado por su énfasis en la interdependencia de todos los elementos naturales, de modo que se creen condiciones para que los niños y las niñas de 6 a 12 años - la ciudadanía mundial del futuro- pue- dan potencializar sus sentimientos de cooperación, respeto y amor en relación con la propia naturaleza y el cosmos. "La vida es un agente cósmico. ¿Cómo esa verdad debe ser presentada a los niños y niñas de manera que toque su imaginación?", se cuestiona María Montessori (2004, p. 32). De forma similar a la gran historia, la educación cósmica se basa en dar libertad a los niños para explorar, estudiar y adquirir conocimientos sobre los inicios del universo, el origen de la vida, la evolución del ser humano, el desarrollo del lenguaje y la historia de las matemáticas. Por todo ello es necesario promover una transformación epistemológica estructural que facilite el desarrollo de un pensamiento complejo capaz de construir un nuevo tipo de identidad para la emergente ciudadanía global. Una identidad global basada en la idea de que los seres humanos somos parte de la naturaleza (gobernados por leyes naturales), cuyo planteamiento histórico aborde el pasado de los pueblos, de la vida, de la Tierra y del universo. Es decir, una perspectiva transdisciplinar, cuyo enfoque dinámico comprenda la complejidad de las relaciones sociales de nuestro tiempo con la naturaleza, en armonía con el marco teórico de la gran historia, donde el ser humano es considerado una parte importante de los procesos coevolutivos.

\section{La creatividad en los procesos coevolutivos de la gran historia}

De acuerdo con el consenso científico actual de la gran historia (Spier, 2011, p. 101), el universo humanamente conocido surgió hace unos 13700 millones de años antes del presente (AP), con la explosión del big bang. La formación de la Tierra se produjo entre 5000 y 4500 millones de años AP, y el milagro de la vida apareció en torno a los 3800 y 3500 millones de años AP. Durante la primera mitad de este periodo las formas de vida primogénitas de la Tierra se mantuvieron en niveles de complejidad muy sencillos (como las arqueobacterias o las eubacterias), pero la aparición del oxígeno libre en la atmósfera originó las primeras células complejas 
(las eucariotas), hace unos 2000 millones de años AP. La explosión cámbrica del metazoo tuvo lugar unos 1500 millones de años después, hace unos 542 millones de años AP. Desde entonces la variedad biológica se ha incrementado a gran velocidad y ha formado una amplia gama de organismos multicelulares que vienen desarrollando estrategias de supervivencia con flujos de energía muy singulares, como la cadena trófica. Si bien todo parece indicar que la vida surgió en las profundidades de los océanos, no consiguió alcanzar la tierra firme hasta hace unos 450 millones de años AP. Tan solo 250 millones de años después de alcanzar la superficie terrestre surgieron los primeros animales de sangre caliente, donde destacaron los dinosaurios del periodo Jurásico que desaparecieron hace unos 66 millones de años AP por el impacto de un supuesto asteroide en la Tierra. Esta circunstancia dio lugar al periodo hegemónico de los mamíferos, de donde emergieron más tarde los primeros homínidos bípedos, en torno a 7 millones de años AP. Gracias a la prueba del carbono 14 realizada en los restos fósiles encontrados hasta la fecha, se puede conocer de un modo aproximado la datación de los primeros australopitecos, que parecen tener unos 4 millones de años AP. Los de Homo habilis datan de 2.5 millones de años AP, los de Homo erectus tienen en torno a 2 millones de años AP y los del Homo neanderthalensis y Homo sapiens apuntan a unos 200000 años AP. Con la extinción del Homo floresiensis hace unos 13000 años AP, el Homo sapiens es el único superviviente de la especie humana que cohabita y coevoluciona en el planeta Tierra junto con el resto de la biodiversidad animal, vegetal, insectos, bacterias, etc.

La coevolución es un concepto acuñado por el biólogo Paul R. Ehrlich y el botánico y ambientalista Peter H. Raven en 1964. En su trabajo conjunto "Butterflies and plants: A study in coevolution", trataron las influencias evolutivas recíprocas que tienen las plantas y los insectos que se alimentan de ellas: "Un abordaje que nos gustaría llamar de coevolución es el examen de los patrones de interac- ción entre dos grandes grupos de organismos con una relación ecológica cerrada evidente, tal como las plantas y los herbívoros [la traducción es mía]" (p. 586). Si bien la idea de coevolución no era nueva y ya se había expresado en teorías anteriores, el uso que Ehrlich y Raven hicieron del término permitió que pensadores de otros campos de aplicación hicieran nuevas interpretaciones. En 1980, el ecólogo evolucionista Daniel $\mathrm{H}$. Janzen fue el primero en definir el concepto de coevolución en su artículo "When is it coevolution?":

La coevolución puede ser útilmente definida como un cambio evolutivo en un rasgo de los individuos de una población en respuesta a un rasgo de los individuos de una segunda población, seguido de una respuesta evolutiva de la segunda población por el cambio en la primera [la traducción es mía]. (p. 611)

Añade que "la coevolución difusa ocurre cuando una o ambas poblaciones en la definición anterior están representadas por una serie de poblaciones que generan una presión selectiva como un grupo" (Janzen, 1980, p. 611). De este modo, la interdependencia ecológica requiere tres principios básicos: 1. especificidad, donde la evolución de cada especie se debe a las presiones selectivas de la otra; 2 . reciprocidad, al evolucionar conjuntamente ambas especies; 3. simultaneidad, donde ambas especies evolucionen al mismo tiempo. Así, el proceso coevolutivo se ha estado usando en un sentido relativamente restricto en la evolución biológica.

Pero el sentido de coevolución que se utiliza para discutirlo en la educación va más allá: abarca e integra tanto el grado de asociación filogenética mutua como el grado de modificación mutua en la coadaptación, así como los procesos de macroevolución y microevolución. La coevolución puede ser definida, entonces, como un cambio evolutivo recíproco entre especies y su entorno natural que, durante el desarrollo complejo de interretroacciones entre sí, se modifican mutuamente de forma 
constante. Esta perspectiva coevolucionista le sirve al investigador Rolf Zinkernagel (2007), Premio Nobel de Medicina de 1996, para explicar cómo el sistema inmunitario ha coevolucionado con microbios que causan enfermedades infecciosas. En términos generales, la coevolución es un proceso de retroalimentación que está muy presente en la naturaleza y que también ha servido de base para la explotación agrícola e industrial del ser humano durante su evolución histórica en la Tierra. Como bien explica el economista ecológico Richard B. Norgaard (1994, p. 39), "con la industrialización, los sistemas sociales coevolucionaron para facilitar el desarrollo a través de la explotación del carbón y del petróleo. Los sistemas sociales ya no coevolucionaron para interactuar más eficazmente con los sistemas ambientales [la traducción es mía]". Con la revolución industrial, se inició una era de hidrocarburos que modificaron drásticamente los procesos coevolutivos de la etapa agrícola anterior del ser humano. En la medida en que los sistemas sociales comenzaron a ejercer fuertes presiones en los sistemas ambientales, el stock de recursos materiales y energéticos disminuyó muy rápidamente y se inició un periodo evolutivo de insostenibilidad planetaria.

La sociedad globalizada del siglo XXI tiene que tomar consciencia, de forma urgente, de la insostenibilidad socioeconómica de la industrialización, cuyos patrones de consumo y producción ponen en riesgo tanto a las generaciones humanas futuras como al resto de los ecosistemas naturales. Es necesario organizar el conocimiento de forma transdisciplinar para comprender que nuestra evolución como especie está intrínsecamente interligada en los procesos de coevolución constante que las distintas formas de vida vienen desarrollando en nuestro planeta Tierra desde hace miles de millones de años. Se trata de una coevolución multidimensional que se desenvuelve a través de interretroacciones entre los diferentes niveles de realidad cósmica, planetaria, regional, nacional y local, donde se establece una extensa red de interdependen- cia universal de fenómenos ecológicos, biofísicos, sociales, políticos, culturales, económicos, tecnológicos, etc. De ahí que la explotación descontrolada de los recursos naturales para la fabricación de productos industriales se haya convertido en una problemática de gran preocupación en la agenda internacional, donde diferentes actores geopolíticos estudian y analizan desde hace décadas los fenómenos transfronterizos que afectan a todas las formas de vida. Es urgente preservar los millones de especies que conforman la creativa biodiversidad de los ecosistemas naturales, pues son un verdadero milagro cósmico. En este sentido, los ODS emergen como un marco de acción transnacional que persigue movilizar a la ciudadanía mundial para tratar con urgencia la enorme huella ecológica y social a la que hemos llegado en el siglo XXI.

\section{¿Qué son los Objetivos de Desarrollo Sostenible (ODS)?}

En septiembre de 2015, la Asamblea General de las Naciones Unidas celebrada en Nueva York consiguió que 193 Estados miembros se comprometieran a ampliar su lucha para frenar la degradación medioambiental con los nuevos ODS para 2030. La Declaración Transforming our world: the 2030 Agenda for Sustainable Development firmada por los líderes mundiales incluye el cambio climático, la conservación de los ecosistemas terrestres, de los mares y océanos, así como otras metas de carácter sistémico y global. En síntesis, los ODS suponen el reconocimiento de problemas socioecológicos que caracterizan a la civilización planetaria actual más allá de sus fronteras nacionales. De ahí la necesidad de transgredir el paradigma actual con el nuevo enfoque que la gran historia nos brinda, puesto que representa una herramienta epistémica que concibe las interrelaciones de la condición humana dentro de su contexto cósmico y terrenal. Se trata de una nueva organización transdisciplinar del conocimiento que nos permite incluir biomiméticamente los sistemas culturales humanos y los ecosistemas 
naturales dentro de un mismo proceso histórico coevolutivo. La gran historia nos ayuda a identificary reconocer las estrategias de sostenibilidad que funcionan en la naturaleza para inspirarnos biomiméticamente en la resolución de distintos problemas humanos (sociales, económicos, tecnológicos, etc.). La continua explotación de los recursos materiales y energéticos de la Tierra por los modelos de producción y consumo ha originado una gran huella ecológica y social que se ha revelado como insostenible. Desde la revolución agrícola de hace unos 10000 años, y especialmente desde la revolución industrial de hace unos 250 años atrás, las acciones del ser humano en el medio ambiente vienen ocasionando un "ecocidio" que está llevando a la extinción a diferentes especies naturales, únicas en su creatividad evolutiva. Por el contrario, una sociedad que camina hacia el desarrollo sostenible tiene que aprender a reducir su destrucción ecológica y reutilizar y reciclar los materiales ya construidos. El desarrollo sostenible es un proceso dinámico que requiere una visión "glocal", ya que el progreso global es una emergencia del sistema planetario nutrida de múltiples progresos locales que avanzan a través de mecanismos sistémicos (retroalimentaciones, sinergias, etc.) que interactúan unos con otros e influyen, condiciona y modifican los diferentes contextos de la ciudadanía mundial. De ahí que tengamos que enfocar nuestra visión en el horizonte paradigmático de los ODS a escala multinivel: planetaria, regional, nacional y local y engendrar un mundo donde "otros mundos sean posibles", lo cual implica reconocer e identificar transculturalmente las estructuras y los fenómenos cósmicos que transcienden paradigmáticamente la condición humana, en armonía con la creatividad potencializada por la educación cósmica del método pedagógico de María Montessori (2004).

$2 \quad$ El término glocal es acuñado por Robertson (1992) y es un neologismo formado por las palabras globalización y localización. Para Robertson (1992), la globalización no implica una anulación de lo local sino una inclusión, presencia y encuentro de y con las culturas locales.
En este proceso de potencialización de la creatividad humana en su formación y modelaje, la biomímesis emerge como una ciencia transdisciplinar que se ocupa de estudiar la complejidad de las interretroacciones desarrolladas entre los sistemas dinámicos que componen la vida (ser humano, animales, plantas, etc.), dentro de un entorno ambiental que alberga las condiciones idóneas para su coevolución. El ser humano es una especie única que participa de una gran danza cósmica protagonizada por fenómenos de energía-materia, cuya sinfonía nos recuerda que somos actores activos de la coevolución del mundo común compartido con los ecosistemas de Gaia. "Reconocemos ahora la Tierra como un ser autocreativo único, que adquirió vida en su danza giratoria por el espacio [la traducción es mía]", expresa la bióloga Elisabet Sahtouris (1998, pp. 25-26) y añade: "En cuanto reunimos los detalles científicos de la danza de la vida de nuestro planeta [...], la evolución de nuestra especie adquiere un nuevo significado en relación con el todo". De ahí que la degradación sistemática de la naturaleza nos convierta en cómplices de un ecocidio globalizado, puesto que la huella ecológica (Wackernagel y Rees, 1996) se perpetúa por nuestra activa participación en dinámicas consumistas y por nuestra pasividad bioética ante la destrucción de la vida en nuestro planeta Tierra, que es nuestro bien común más sagrado. "Hay pocos indicadores más alarmantes acerca del brutal desequilibrio climático que hemos puesto en marcha, y cuyas consecuencias serán terribles (ecocidio más genocidio, si se quiere expresar en una fórmula sintética)" expresa el filósofo Jorge Riechmann (2014, p. 24). Con tales desequilibrios, las generaciones futuras sufrirán las consecuencias climáticas de un calentamiento global provocado por nuestra cultura de consumo y producción actual (escasez crónica de recursos, cambios ecosistémicos, pérdida de la biodiversidad, deshielo glaciar, aumento del nivel del mar, deforestación, contaminación del suelo, agua y aire, etc.). Por esta razón, la biomímesis representa un verdadero salto cualitativo en la construcción epistemológica del conocimiento 
para caminar a la sostenibilidad requerida por los ODS, puesto que su marco epistémico multirreferencial va más allá de las problemáticas morales tradicionales del bienestar humano para integrar nuevos avances y desarrollos tecnológicos que modifican radicalmente los fenómenos vitales de la propia naturaleza.

Desde esta visión cosmoderna, propongo que el debate sobre los ODS no se debe enfocar en encontrar solución a los problemas cada vez más complejos que surgen del actual sistema de referencia económico de la sociedad-mundo del tercer milenio. Los ODS deben promover la transformación del propio sistema de producción capitalista e inspirarse en el abordaje biomimético. Afirmar que el crecimiento económico es bueno por sí mismo, postulando que los niveles de calidad humana se miden por el PIB (producto interior bruto) y el PNB (producto nacional bruto) de un país, supone cometer un fraude intelectual de peligrosas consecuencias en la era de la crisis ecológica global. Si bien es cierto que el sistema capitalista ha traído enormes beneficios materiales, su visión funcionalista subordina todo al máximo beneficio económico y al consumo indiscriminado en detrimento de la naturaleza. No se trata de debatir entre comunismo, anarquismo, socialismo, capitalismo o cualquier teoría política de organización social derivada de estructuras mentales mecanicistas, sino de imitar a la propia naturaleza: "Si queremos llevarnos bien con Gaia, es justamente así como tenemos que vernos a nosotros mismos, como un voto en un parlamento de treinta (o quizás hasta cien) millones de escaños, una especie entre especies" (Benyus, 2012, p. 24). ¿Por qué la especie humana continúa hipotecando el futuro de millones de especies por su absurda lógica de consumo irracional que implica la explotación de los recursos naturales? ¿Por qué creemos en la ilusión epistemológica de un crecimiento económico ilimitado cuando nunca ha existido en la naturaleza especie viva alguna que creciera sin cesar hasta el infinito? ¿Sería posible crear nuevos horizontes civilizatorios inspirados por la creatividad intrínseca en el ADN de los procesos coevolutivos de la naturaleza? ¿Cómo se podría enriquecer la formación humana con la aplicación biomimética de la sabiduría sostenible de la naturaleza?

\section{Biomímesis: ¿ modelo educativo para desarrollar una formación humana sostenible?}

El enfoque biomimético es una de las respuestas más innovadoras de los últimos años para proteger el medio ambiente y mejorar la calidad de vida a través de nuevos hábitos de consumo y producción sostenibles. El término biomímesis proviene del griego bios, vida, y mímēsis, imitación. En la década de 1990, el término biomímesis sería usado en ámbitos disciplinarios de las ciencias materiales, la investigación cosmética y la robótica, hasta que la escritora de ciencias naturales estadounidense Janine M. Benyus lo popularizaría con su libro Biomimicry: Innovation inspired by nature. Desde entonces, la biomímesis se erigió como una nueva ciencia que contempla y valora la naturaleza como modelo, medida y mentor en busca de la inspiración e imitación de los procesos naturales para aplicarlos a sistemas sociales y de este modo encontrar soluciones innovadoras a problemas complejos (como los ODS). "La biomímesis se vale de un estándar ecológico para juzgar la corrección de nuestras innovaciones. Después de 3800 millones de años de evolución, la naturaleza ha descubierto lo que funciona, lo que es apropiado y lo que perdura" señala Benyus (2012, p. 13) y afirma que la biomímesis "inicia una era basada no en lo que podemos extraer del mundo natural, sino en lo que éste puede enseñarnos". La biomímesis representa la unión teórico-práctica entre el Norte y el Sur, además de una herramienta epistémica creativa para combatir los desafíos del cambio climático, que es el peligro más urgente en la actualidad. En esta línea de pensamiento, Benyus identifica nueve principios operacionales básicos que la vida desarrolla en la naturaleza que pueden ser usados 
en la formación humana requerida por la educación para el desarrollo sostenible y la educación para la ciudadanía mundial, articuladas en el punto 4.7 de la estructura general de los ODS. Estos principios de sostenibilidad biomimética son los siguientes:

1. La naturaleza cabalga sobre la luz solar: la energía que absorbemos casi todas las comunidades naturales proviene de la fusión nuclear que el Sol realiza a 150 millones de kilómetros. "Las energías solar, eólica y mareal, así como el biodiésel, derivan todas de la luz solar actual" (Benyus, 2012, p. 321). Cuando quemamos restos fósiles, como petróleo, gas natural o carbón, estamos usando la luz solar antigua que quedó atrapada (comprimida en un medio sin oxígeno) en los cuerpos de animales y plantas del periodo Carbonífero. Al realizar la combustión, estamos completando "el proceso de descomposición de golpe, vertiendo el carbono almacenado a la atmósfera en grandes cantidades, y desoyendo así el precepto ecosistémico de nada de flujos grandes" (p. 321). Teniendo en cuenta que la bioesfera Gaia es un sistema cerrado y autopoiético, esa actitud equivaldría a quemar los muebles dentro de nuestra casa con las ventanas cerradas. Por desgracia, los combustibles fósiles son demasiado baratos y la sociedad actual de consumo, adicta a la energía, se dirige a la explotación total de estos recursos naturales. Un buen ejemplo serían las hojas, que realizan la fotosíntesis (descomposición bioquímica de la energía solar en nutrientes) "con una eficiencia cuántica de un asombroso 95 \%" (Benyus, 2012, p. 319), más de cuatro veces la de los mejores paneles solares de construcción humana.

2. La naturaleza gasta solo la energía que necesita: si bien es cierto que la segunda ley de la termodinámica convierte la energía en calor, y una parte de energía deja de ser aprovechable, la naturaleza sabe cómo obtener energía de forma eficiente a través de diversas conexiones ecosistémicas. Con el fin de hacer un uso óptimo del hábitat limitado, cada organismo encuentra un nicho y tan solo usa lo que necesita para sobrevivir y evolucionar. De este modo, las lecciones de los sistemas naturales pueden orientarnos a establecer nuevos usos para la energía. Debemos reconsiderar lo que estamos maximizando (la producción) y fijarnos más en la optimización, tal y como hacen los sistemas naturales, que invierten su energía en maximizar la diversidad para hacerse más eficientes en cuanto al reciclaje de nutrientes orgánicos y minerales (Benyus, 2012, p. 322).

3. La naturaleza ajusta la forma a la función: la naturaleza constituye un sistema altamente cooperativo constituido por densas interacciones entres sus componentes. Toda la red ecosistémica ha sido construida en los límites de los recursos disponibles $y$, como resultado de ello, el sistema entero ha alcanzado una coherencia interna de intrincados patrones orgánicos, cuyo tamaño se adapta a la función. La naturaleza optimiza en lugar de maximizar. Por el contrario, nuestros ecosistemas industriales "siguen apostando por tasas elevadas de productividad y crecimiento, por un caudal máximo de materiales extraídos de la tierra y convertidos en flamantes artículos nuevos. El 85 \% de los artículos manufacturados se convierte rápidamente en basura" (Benyus, 2012, p. 323). En efecto, la economía globalizadora actual define su éxito por el rápido crecimiento y crea la ilusión medir el progreso y desarrollo humano por índices como el PIB y el PIN (producto interior neto). Por el contrario, los organismos coevolucionan en la naturaleza y se adaptan a los cambios de los demás; es decir, haciendo que una estructura desempeñe no una sino varias funciones en su entorno. "La lección es que tenemos que retardar la transformación de materiales y poner el énfasis en la calidad y no en la cantidad de artículos nuevos" (Benyus, 2012, p. 323).

4. La naturaleza lo recicla todo: "Una de las lecciones clave de la ecología de sistemas es que a medida que un sistema acumula biomasa (peso total de materia viva), necesita más reciclaje para eludir el colapso" (Benyus, 2012, p. 312). La existencia de cadenas tróficas en los ecosistemas tiene un esquema organizativo circular donde los productores, consumidores y descomponedores han evolucionado conjunta- 
mente a un bucle cerrado para impedir la pérdida de recursos: "todo desecho es alimento, y todo el mundo se reencarna en el cuerpo de otro" (p. 313). El problema de la cultura humana de producción y consumo es que continúa acumulando biomasa sin una red de bucles cerrados. En este sentido, Benyus (2012) explica varios ejemplos de "economía sin desechos" en los países nórdicos europeos (especialmente Dinamarca) en los que existen pequeñas redes tróficas de ecología industrial con bucles cerrados y el intercambio de información y el deseo mutuo de aprovechar los desechos promueve que todos los productos que salen manufacturados al mercado vuelvan a entrar en el sistema de producción a través de leyes de recuperación y sistemas de reembolso.

5. La naturaleza premia la cooperación: en los ecosistemas maduros, las estrategias cooperativas entre los organismos son tan importantes como la competencia. De acuerdo con la hipótesis de endosimbiosis de Lynn Margulis (2002), la simbiosis entre dos especies es un elemento fundamental del progreso evolutivo natural desde hace miles de millones de años. Los ecosistemas naturales operan en una compleja red simbiótica de relaciones mutuamente beneficiosas y, cuando se agrupan en gran número, constituyen órganos y organismos. De hecho, la teoría endosimbiótica postula que nuestro cuerpo es en realidad una combinación de organismos unicelulares que han conformado un enorme organismo pluricelular. Traducido al sistema de producción humana, el ecólogo industrial japonés Michiyuki Uenohara (2012), señala:

Tenemos arterias (vías por las que fluyen los productos desde el corazón industrial hasta el cuerpo de la economía) de sobra, pero también necesitamos venas, vías de retorno de los productos usados para que sus materiales puedan purificarse y reutilizarse. (citado por Benyus, 2012, p. 318).

La lección aprendida, por tanto, es construir una economía donde las arterias y las venas tengan la misma importancia, lo cual conllevaría la imitación de una ecología de sistemas de bucle cerrado que reutiliza los recursos.

6. La naturaleza cuenta con la diversidad: el enorme desarrollo de la diversidad de la naturaleza se debe a su experiencia de miles de millones de años en ensayo y error. La naturaleza se caracteriza, en consecuencia, por el enfoque multirreferencial que la aleatoriedad producida por la entropía (ruptura del orden) ha permitido con su gran abertura flexible a nuevas anomalías. Esta flexibilidad ecobiológica ha permitido una gran variedad de animales y plantas a lo largo de miles de millones de años en torno de todo el hábitat del planeta Tierra. Por tanto, la lección que aprendemos de la naturaleza es que nuestro sistema industrial debe ser flexible para adaptarse a las necesidades emergentes de la ciudadanía global, y ser tan diverso como su propio entorno para respetar la singularidad regional, cultural y material del lugar.

7. La naturaleza demanda tecnología local: generalmente, los ecosistemas naturales están conectados de manera relativamente cercana en el espacio-tiempo. Existe una rica biodiversidad en los ecosistemas locales donde muchas especies locales coevolucionan conjuntamente para adaptarse a los cambios. Pero la tendencia capitalista actual es una economía global sin fronteras donde los productos manufacturados se elaboran en países muy separados geográficamente. En este sentido, debemos aprender de la experiencia y del conocimiento local que los pueblos indígenas poseen, ya que "la idea de una economía que se adecúe a la tierra y saque partido de sus atributos locales nos acercaría más a los organismos que han evolucionado para convertirse en expertos locales" (Benyus, 2012, p. 339).

8. La naturaleza frena los excesos desde dentro: "La biosfera (la capa de aire, tierra y agua que sustenta la vida) es un sistema cerrado, lo que significa que no se importan ni exportan materiales (aparte de los traviesos meteoritos)" (Benyus, 2012, p. 332). El 
carácter autopoiético (Maturana y Varela, 2001) de la biosfera consigue que la vida mantenga las condiciones que le son necesarias para autorregularse a través de un incesante intercambio entre organismos (fotosíntesis, respiración, crecimiento, mineralización, descomposición, etc.). Por desgracia, el sistema industrial global es un sistema abierto en el que los "nutrientes" se transforman en "desechos", sin que haya un reciclaje significativo. Esta dinámica de explotación de los recursos naturales y contaminación está cambiando drásticamente los procesos naturales, porque no pueden reciclar las enormes cantidades de $\mathrm{CO}_{2}$ vertidas a la atmósfera (actualmente 355 de cada millón de moléculas). La única respuesta es un ecosistema industrial que pueda integrarse en la biosfera sin dañarla.

9. La naturaleza saca partido de las limitaciones: la naturaleza ha aprendido que vivir con los recursos finitos es una poderosa fuente de creatividad. En la naturaleza, hay mecanismos de retroalimentación interna que optimizan el uso de los recursos del entorno en constante balanza, con moderación y sin devastarlo. Eso significa no hipotecar el futuro, dado que, de lo contrario, moriría. La lección es que nuestro sistema productivo actual no puede continuar empujando los límites del planeta. La naturaleza nos enseña a florecer dentro de los límites biológicos, sin estar en continua expansión depredadora. Por el contario, debemos "adaptar los sistemas humanos a los ecosistemas (biomímesis), lograr mayores eficiencias (ecoeficiencia) y actuar sobre la demanda con medidas de autocontención (gestión generalizada de la demanda)" (Riechmann, 2014, p. 28).

Estos nueve principios operacionales básicos que la vida desarrolla en la naturaleza, y que Benyus (2012) identifica muy acertadamente, son incompatibles con el orden socioeconómico capitalista actual.

Podría decirse incluso que el capitalismo es la antitesis metafórica de los procesos naturales de la vida: en él priman la exclusión, el despilfarro, la desregulación y las hoy llamadas des- localizaciones, asi como los flujos especulativos ajenos a la producción real de bienes y servicios [en comparación a los sistemas naturales de la biosfera donde] operan circuitos incluyentes de todos los miembros de la red, los cuales están apegados al terreno, ligados a la satisfacción de las necesidades básicas y al reciclado constante de materia y energía. (Espinosa, 2007, p. 66)

Esta comparación supone, efectivamente, la comprensión ecoética de la propia vida en su complejidad multidimensional. Una comprensión ecoética que debe ser promocionada por la Educación para la ciudadanía mundial (ECM) para hacer frente a las dinámicas tecno-economicistas globalizadoras que están acabando con la vida en el planeta. La ECM debe aspirar a constituirse como la herramienta política, educativa y epistemológica capaz de modificar el metabolismo socioecológico a través de nuevas simbiosis entre los ecosistemas naturales y los sistemas culturales humanos de producción. Para ello, debemos tratar el principio de biomímesis en un sentido más amplio, con el fin de "comprender los principios de funcionamiento de la vida en sus diferentes niveles (y en particular en el nivel ecosistémico) con el objetivo de reconstruir los sistemas humanos de manera que encajen armoniosamente en los sistemas naturales" (Riechmann, 2014, p. 171).

Dicho en otras palabras, lo que Riechmann (2014) entiende por reconstruir los sistemas humanos significa dejar de crecer económicamente para enfocarnos más en el desarrollo cualitativo. Del mismo modo que no existe especie viva en la naturaleza que crezca todo el tiempo, la economía (como eje transversal de los sistemas humanos) debe estacionarse, consumir solo los recursos naturales necesarios y centrarse en las capacidades humanas de forma ampliada. Esto significa que los procesos de enseñanza-aprendizaje educativos deben suscitar un diálogo biomimético que fomente una consciencia crítica planetaria mediante reflexiones solidarias que, en última instancia, favorezcan la aparición de nuevas propuestas de organización 
social para el cumplimiento de los ODS. Así, la educación actuaría como una estructura orgánica viva en constante proceso de adaptación y coevolución con el medio, pues la educación para la sostenibilidad no solo deberá pensar en cómo integrar los principios biomiméticos en las estructuras políticas y los currículos educativos nacionales/regionales/ locales, sino que también deberá pensar en cómo aplicarlo desde el punto de vista de las redes. Dado que el microcosmos escolar encarna el macrocosmos de las estructuras sociales, el futuro común de la humanidad y del planeta Tierra requiere una auténtica transformación política, epistemológica y educativa, que suponga la emergencia de un nuevo paradigma civilizatorio caracterizado por el cambio de jerarquías a redes en la organización social. De ahí que sea fundamental una formación humana transdisciplinar para conseguir enfrentarnos a los desafíos de insostenibilidad planetaria antes de que sea demasiado tarde.

\section{Educar para vivir en la cosmodernidad}

En esta línea de pensamiento transdisciplinar, se encuentran los modelos de formación humana planteados por el psicólogo y científico de la educación Gaston Pineau (2004) y el médico y antropólogo Patrick Paul (2009). En ambos modelos teóricos de formación humana, se busca pensar de manera compleja para entender las interrelaciones del todo con las partes, y viceversa. En consecuencia, el conocimiento y el aprendizaje humano implican el desarrollo de procesos autorreguladores, autoorganizadores y autotransformadores que envuelven diferentes dimensiones presentes en la complejidad humana. De acuerdo con la teoría tripolar de la formación postulada por Pineau (2004), en la que se explora la metodología de las historias de vida y las diversas formulaciones que los sujetos dan para sus trayectos formativos, existen tres procesos esenciales en la formación humana: 1. la personalización, 2. la socialización y 3 . la ecologización. Esta perspectiva teórica le llevó a formular tres conceptos de forma- ción humana: 1. la autoformación, en relación con uno mismo; la heteroformación, en relación con las otras personas; y la ecoformación, en relación con el mundo. Según explica Pineau (2004, p. 130), el término autoformación surgió antes que los otros dos y favoreció el desarrollo de investigaciones sobre "el empoderamiento de los actores por la apropiación de su poder de formación". El concepto de heteroformación se refiere a la dimensión social de la acción educativa y formativa entre las demás personas y el término de ecoformación designa los procesos formativos con respecto al medio ambiente material (Pineau, 2004, p. 132). A su vez, resalta que ninguna de estas dimensiones formativas debe ser priorizada en detrimento de otra, por lo que sugiere el término de coformación para describir ciertos procesos educativos y formativos enfocados en las interrelaciones de los actores, donde se producen interretroacciones no jerárquicas. Es aquí donde Paul (2009) aboga por una articulación de todas estas dimensiones de la persona postuladas por Pineau (2004), para poder desarrollar una cuarta dimensión que denomina ontoformación. De acuerdo con la teoría de la antropoformación de Paul (2009, p. 28), la formación humana es "el proceso global y general (al mismo tiempo particular y singular, pero también social y colectivo) que articula las relaciones interactivas entre ecoformación, heteroformación, autoformación y ontoformación". Además de acrecentar una nueva dimensión, también propone una modelización articulada de los diferentes niveles de realidad formativos del sujeto transdisciplinar, que se sintetizan en la figura 1.

Como Sommerman (2012) sintetiza en la figura 1, la modelización propuesta por Paul es constituida por cuatro dimensiones de la formación humana: 1. la ontoformación (No), la autoformación (N1), la heteroformación (N2) y la ecoformación (N3). Para Paul (2009, pp. 531-535), el nivel de realidad No es unitivo y corresponde a la dimensión de la ontoformación, donde es necesaria una lógica unaria para comprender la virtualidad y la potencialidad que va 
Figura 1. Modelización de la teoria antropoformativa de Paul.

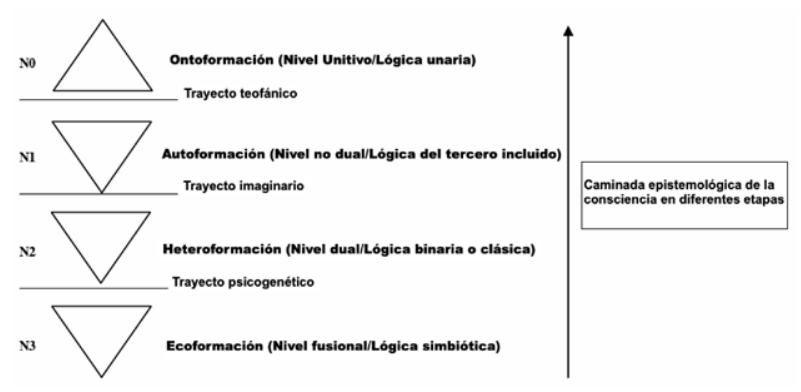

Fuente: Sommerman (2012, p. 808)

más allá de toda forma e imagen de este nivel. El N1 es un nivel no dual que corresponde a la dimensión autoformativa, donde se manifiestan las potencialidades contenidas en el No, para cuya comprensión se requiere la lógica del tercero incluido. El nivel N2 concierne a las interacciones duales de la dimensión heteroformativa, cuya lógica binaria discurre sobre la vida y la muerte, lo subjetivo y lo objetivo, lo individual y lo colectivo, etc. Por último, el nivel fusional N3 corresponde a la dimensión ecoformativa, donde la simbiosis constituye la base funcional de los sistemas vivos de la naturaleza. A su vez, estas cuatro dimensiones son recorridas epistemológicamente por el sujeto transdisciplinar mediante diferentes etapas: pasar de la ecoformación (N3) a la heteroformación (N2) constituye el trayecto psicogenético de la formación del ser humano; el paso de la heteroformación (N2) a la autoformación (N1) constituye el trayecto imaginario; y el pasaje de la autoformación (N1) a la ontoformación (No) constituye el trayecto teofánico de la formación del ser humano global (Paul, 2009, p. 541). En su conjunto, la modelización multidimensional propuesta por Pineau (2004) y Paul (2009) para la formación del ser humano supone un nuevo abordaje transdisciplinar que nos ayuda a afrontar los desafíos planetarios que la humanidad tiene para alcanzar los ODS. Por tanto, educar para vivir en la cosmodernidad significa desarrollar el potencial de las cuatro dimensiones propuestas por Pineau (2004) y Paul (2009) para la formación transdisciplinar de un ser humano complejo en constante coevolución material, energética e informacional.

En este marco de complejidad epistemológica de la formación educativa humana, la educadora ambiental Dominique Cottereau (2001, 2005) complementa la dimensión ecoformadora proponiendo un "vals en tres tiempos para la formación ecológica". Se trata de un diálogo epistemológico que integra las relaciones del ser humano con la naturaleza para estructurar una visión educativa transdisciplinar que promueve un desarrollo sostenible perdurable. "La ecoformación es un proyecto de educación/formación a la altura que los desafíos psicosocioambientales han levantado. Las contribuciones de la exploración conceptual no son sino necesidad de abrir vías de acción para la intervención junto a niños, jóvenes y adultos [la traducción es mía]" (Cottereau, 2005, p. 112). El primer tiempo de la ecoformación concierne al aprendizaje de saberes relativos al medio ambiente, las ciencias naturales y las ciencias humanas que cultivan la razón y el conocimiento. El segundo tiempo enriquece al primero mediante la experiencia práctica del mundo, donde el sujeto transdisciplinar desarrolla lazos afectivos y emocionales con la naturaleza. El tercer tiempo corresponde a la aprensión de la experiencia, de la escucha sensible, la reflexión sobre los gestos normalmente automáticos de nuestra cotidianidad, puesto que la toma de consciencia ecológica implica la retroacción de las acciones autónomas que realizamos de forma casi automática. Salir de la "inconsciencia ecológica" requiere tomar consciencia de las múltiples dependencias que tenemos con el medio ambiente a través de la exploración de nuestras historias, que "hablan sobre nuestra manera de habitar el mundo, de nuestras relaciones con el espacio, con los paisajes, con los objetos, los materiales, con la naturaleza, con las estaciones, con los momentos del día" (Cottereau, 2001, p. 65). En este sentido, Cottereau demanda un papel más relevante de la educación informal y no formal en la ecoformación, dado que esta "gramática de la intuición, de la escucha y 
de lo sensible [...] se enseña bien lejos de los cuadros negros, de los libros de leyes y de las cátedras universitarias [la traducción es mía]" (p. 66). De un modo complementario a esta demanda ecoformativa, el educador Galvani (2001) considera fundamental un intercambio intercultural y transcultural con todas las culturas antiguas, especialmente con los pueblos indígenas originarios de las Américas, puesto que la esencia primordial del acto educativo es la inmersión experimental del sujeto transdisciplinar. El aprendizaje significativo se establece a partir de un "tipo de comportamiento y de valores que se desarrollan en el corazón de la experiencia por medio de una relación global con los otros y con el mundo [la traducción es mía]" (p. 92). Por esta razón, Galvani (2002) define la formación como la historia de las interacciones y de los acoplamientos estructurales del ser humano con su medio ambiente físico y social. De ahí que los diferentes niveles de formación comporten diferentes niveles de interacciones entre las personas y el medio ambiente: "El nivel práctico del gesto, el nivel simbólico del imaginario y el nivel epistémico del concepto" (pp. 102-103). El nivel de interacciones prácticas corresponde a una razón experiencial, el nivel de interacciones simbólicas a una razón sensible y el nivel de las interacciones epistémicas a una razón formal. En suma, el intercambio intercultural y transcultural propuesto por Galvani (2001) para el desarrollo de la ecoformación está en armonía con el concepto de antropoformación creado por Paul (2009) para incluir y articular todas las dimensiones humanas en el trayecto educativo.

Estas propuestas de formación humana logran armonizar una "ecología de inteligencias" donde convergen la teoría de las inteligencias múltiples del psicólogo Howard Gardner (1983), la teoría de la inteligencia emocional formulada por el psicólogo y periodista científico Daniel Goleman (1995) y la inteligencia espiritual postulada por la física y filósofa Danah Zohar con su marido Ian Marshall (2004). Esta ecología de inteligencias humanas consigue reducir el exceso de autoestima del superego e in- tegrar un conjunto de saberes y sentimientos interligados (insights) que desarrollan la aventura de introspección humana en actos de autorreflexión y autoconsciencia. Según Goleman (1995, p. 60), la autoconsciencia es una "permanente atención a lo que estamos sintiendo internamente. En esa consciencia autorreflexiva, la mente observa e investiga lo que está siendo vivenciado, incluso las emociones" [la traducción es mía]. Este tipo de consciencia es similar al concepto de escuch a fluctuante que el famoso neurólogo Sigmund Freud recomendó a aquellas personas que querían dedicarse al psicoanálisis y a la noción de meditación autoconocedora del filósofo y teólogo Jiddu Krishnamurti. En su conjunto, todos estos autores nos incitan a desarrollar un diálogo interior para conocernos a nosotros mismos mediante una experimentación autoconocedora que implica la observación de nuestro propio pensamiento. Investigar la experiencia a través de la introspección a nuestro propio pensamiento requiere reconocer el papel desempeñado por la consciencia. Es evidente que el autoconocimiento no puede ser objeto de una técnica transmisible en sus procedimientos operacionales. De acuerdo con Krishnamurti (1966), es necesaria una idea de luz propia donde el autoconocimiento es un medio metodológico para la práctica investigativa de sí mismo, comprendiendo esa práctica como la más urgente y vital del ser humano para enfrentarse a la profunda crisis ecológica y existencial que vivencia.

\section{Reflexiones abiertas para crear lazos entre la educación y la sostenibilidad}

Como se ha venido debatiendo y reflexionando a largo del trabajo, los desafíos concernientes a los ODS tienen muchos cuestionamientos y abordajes educativos. De ahí la importancia que tiene la dimensión creativa en la formación humana para enfrentarse a la insostenibilidad imperante que la globalización económica neoliberal impone en todas las esferas sociales, ambientales y mentales. No cabe duda de que la educación tiene un papel fun- 
damental para alcanzar un desarrollo sostenible en Gaia: es la semilla que debemos cultivar para nuestro florecimiento presente y futuro. Se trata de una herramienta de transformación epistémica y socioecológica que la Unesco (2002, p. 7) reconoce desde su propia constitución en 1945, donde se declara

que la amplia difusión de la cultura y la educación de la humanidad para la justicia, la libertad y la paz son indispensables a la dignidad del hombre y constituyen un deber sagrado que todas las naciones han de cumplir con un espiritu de responsabilidad y de ayuda mutua.

Desde entonces, los numerosos eventos, congresos, foros y conferencias que la Unesco viene realizando con sus socios han servido para ampliar, desarrollar y concretar la esfera educativa desde diferentes abordajes antropocéntricos y ecocéntricos. Pasados más de setenta años desde su constitución, la Unesco es un referente mundial que promueve el desarrollo humano sostenible a partir de la educación, la ciencia y la cultura. De ahí que los Estados miembros hayan asignado a la Unesco para liderar y coordinar la agenda de la educación 2030 que está dentro de la estructura general de los ODS. Pero conseguir un desarrollo sostenible perdurable significa ir más allá del cumplimiento de los ODS para 2030, por lo que se hace necesaria una visión integral que incluya al ser humano y a su entorno natural dentro de procesos coevolutivos de la gran historia que se vienen describiendo. "La sostenibilidad no es solo un problema entre nosotros los seres humanos", explica la educadora ambiental María Novo (2009, p. 368), sino que "es también un problema gravísimo de nuestras relaciones con la biosfera, de la forma en que nos apropiamos de los recursos, explotamos la naturaleza, gestionamos los bienes comunes, consideramos los límites de los ecosistemas". Por esta razón, el horizonte de la sostenibilidad global solo será posible si logramos reorientar nuestros modelos de vida dentro de los límites biofísicos de la naturaleza, sin comprometer su regeneración ecosistémica ni el desarrollo digno de nuestras próximas generaciones. Es urgente transformar los modelos y los comportamientos depredadores que la especie humana ejerce sobre Gaia, así como los procesos desiguales de distribución de la riqueza que tan solo benefician a una minoría de la ciudadanía mundial. En este sentido, la identificación de los principios operacionales y las estrategias que la vida viene desarrollando en la naturaleza, desde hace unos 3800 millones de años, supone modelos pedagógicos biomiméticos que nos ayudan a educar para vivir en la cosmodernidad: donde el ser humano coevoluciona en armonía sostenible y resiliente con los ecosistemas de Gaia.

Educar para vivir en la cosmodernidad significa introducir abordajes transdisciplinares y biomiméticos en todos los niveles de la educación formal, pero también en los ámbitos no formales e informales que también sirven para educar el potencial de la condición humana (Collado, 2016c). Desde su aparición en la Tierra hace unos 200000 años atrás, el ser humano moderno ha tenido que aprender a cooperar para sobrevivir física, mental, espiritual y emocionalmente, por lo que ha tenido que aprender durante su camino evolutivo a compartir alimentos, cuidar de sus predecesores, convivir sexualmente, etc. Estas interacciones sociales primitivas se vieron favorecidas por la aparición del lenguaje, que dio lugar a un espacio conversacional de conductas consensuadas para la aceptación mutua. Durante la mayor parte de la historia evolutiva del ser humano, la educación no formal ha sido la predominante en todos los pueblos de la Tierra. En la actualidad, multitud de pueblos indígenas originarios todavía continúan formando a las personas a través de una "mirada bioalfabetizadora" que perdura desde hace miles de años. Lejos de educarlos para ser trabajadores sumisos de un sistema económico globalizador que tiende hacia la homogeneización de la diversidad cultural y que acaba con gran parte de la biodiversidad de Gaia, la formación humana que se desarrolla entre los pueblos indígenas y aborígenes originarios está enfocada en fortalecer los vínculos 
y las relaciones entre el ser humano y la naturaleza. De ahí que la Declaración de la Naciones Unidas sobre los Derechos de los Pueblos Indígenas (2007, p. 2) reconozca "que el respeto de los conocimientos, las culturas y las prácticas tradicionales indígenas contribuye al desarrollo sostenible y equitativo y a la ordenación adecuada del medio ambiente". En su conjunto, las cosmovisiones de los pueblos originarios son un buen ejemplo de desarrollo sostenible y resiliente, tanto por sus prácticas socioecológicas como por su largo recorrido histórico practicándolas. Si bien es cierto que no se puede idealizar a los pueblos indígenas originarios, porque algunos también llevan a cabo prácticas ecocidas, su rica multirreferencialidad epistémica está en plena armonía con los límites y márgenes coevolutivos que los ecosistemas naturales establecen de forma autoorganizativa. Por este motivo, muchos saberes ancestrales representan un buen ejemplo de praxis biomimética (Collado, 2016b).

Como es evidente, todas las ideas transdisciplinares y biomiméticas de la investigación tienen que ser comprendidas como un proceso creativo en continua evolución y construcción, abierto a nuevas interpretaciones, complementaciones y consideraciones. Si bien no existen fórmulas pedagógicas mágicas que solucionen los complejos problemas de nuestro mundo desde una participación social activa, no cabe duda de que debemos enfrentar la encrucijada paradigmática del cambio climático desde una "ecología de saberes" (Santos, 2010) que desarrolle y potencialice todas las dimensiones humanas a través de una organización transdisciplinar del conocimiento que combine la razón científica con otros aspectos cognitivos, intelectuales, perceptivos, afectivos, emocionales, espirituales, religiosos, políticos, retóricos, poéticos, artísticos, epistémicos y filosóficos del ser humano.

En suma, todas estas cosmovisiones y construcciones epistemológicas nos ayudan a enfrentarnos a un reto de gobernabilidad global sin precedentes históricos, donde la solidaridad y la cooperación hu- mana es la clave simbiótica para integrar la bioética como metapunto de encuentro civilizatorio. De ahí que esta investigación persiga construir una gran familia humana a través de una consciencia cosmoderna que identifique la condición/identidad en la Tierra-Patria con el estudio de la gran historia, donde la nueva perspectiva biomimética nos ayuda a reforzar los lazos entre la educación y la sostenibilidad mediante la modelación de la formación humana en relación con el mundo (ecoformación), con otras personas (hetero-y coformación), consigo mismo (autoformación) y como ser (ontoformación). Además, reforzar los lazos entre la educación y la sostenibilidad también significa implementar una visión bioinspiradora y creativa en los contenidos pedagógicos de la escuela y de la universidad para crear nuevos modelos socioeconómicos de carácter planetario en armonía ecológica con la naturaleza. La biomímesis es un punto de encuentro entre las sociedades denominadas primitivas y las llamadas hipertecnológicas, ya que alberga una ecología de saberes científicos y no científicos que desempeña el papel simbiogenético entre la naturaleza y la cultura humana. Según la antropóloga y economista mexicana Cristina Núñez (2012, p. 109), "la experiencia educativa transdisciplinar para la sostenibilidad incluye la dimensión espiritual como un núcleo para la creación relevante en nuestras sociedades en el ámbito local y mundial [la traducción es mía]". Siendo así, es necesario combinar un marco de convergencia entre el conocimiento científico que el universo físico exterior nos ofrece y el conocimiento espiritual del universo emocional interior del género humano (Krishnamurti, 1966). De acuerdo con Núñez (2012), las tradiciones filosóficas ancestrales de los pueblos indígenas originarios nos muestran que las experiencias psicosomáticas entre el cuerpo y la mente nos ayudan a establecer y desarrollar conexiones sacras entre la naturaleza y la vida que promueven hábitos y prácticas socioeconómicas humanas sostenibles con el medio ambiente. Otro buen ejemplo contemporáneo que procura rescatar de forma creativa esos conocimientos milenarios de 
ISSN 0123-1294 | e-ISSN 2027-5358 | Educ.Educ. Vol. 20. No. 2 | Mayo-agosto de 2017 | pp. 229-248.

Universidad de La Sabana | Facultad de Educación

los pueblos aborígenes de Australia es la permacultura creada por el científico David Holmgren (2010).

Para acabar, hay que reflexionar que es necesario entrever el futuro para estar preparados para cuando llegue, pues no existe duda de que los computadores cuánticos, la inteligencia artificial, la nanotecnología, los lentes de contacto con acceso a internet, la mutación genética del ADN y los viajes en el espacio modificarán radicalmente nuestros hábitos en un corto periodo y contextualizarán al género humano en el paradigma de la cosmodernidad (Collado, Galeffi y Ponczek, 2014). Hacer frente a los desafíos de los ODS requiere crear una ecología de saberes que reintegre los diferentes conocimientos y dimensiones humanas que hemos explicado, dado que la potencialización de la creatividad humana es esencial para enfrentarse a la enorme huella ecológica y social que estamos dejando en Gaia. Esta perspectiva biomimética educativa busca, por tanto, desarrollar nuestra capacidad creativa para luchar por un mundo donde el continuum de la vida persista en su camino evolutivo. ¿Están preparados/as? Se anima a los lectores y lectoras a seguir adelante con cualquier pensamiento bioinspirado por las reflexiones presentadas en esta investigación para la formación educativa humana.

\section{Referencias}

Benyus, J. (2012). Biomímesis: cómo la ciencia innova inspirándose en la naturaleza. Barcelona:Tusquets.

Christian, D. (2010). Mapas del tiempo: introducción a la gran historia. Barcelona: Crítica.

Collado Ruano, J., Galeffi, D. A. y Poncze, R. L. I. (2014). O paradigma da cosmodernidad: uma abordagem transdisciplinar à Educação para a Cidadania Global proposta pela Unesco. Revista da FAEEBA: educação e contemporaneidade, 23(42), 141-152.

Collado Ruano, J. (2016a). Transdisciplinary perspectives in bioethics: A co-evolutionary introduction from the big history. Philosophy and Cosmology, 17, 52-63.

Collado Ruano, J. (2016b). Paradigmas epistemológicos en filosofía, ciencia y educación: ensayos cosmodernos. Saarbrücken: Editorial Académica Española.

Collado Ruano, J. (2016c). Cosmodern education in the sustainable development goals: A transdisciplinary and biomimetic approach from the big history. TheATLAS, 6, 98-122.

Cottereau, D. (2001). Pour une formation écologique: complémentarité des logiques de formation. Éducation permanente, $148,57-67$.

Cottereau, D. (2005). Écoformation, entre soi et le monde. Éducation à l'environnement: de soi au monde, $187,111-117$.

Ehrlich, P. R. y Raven, P. H. (1964). Butterflies and plants: A study in coevolution. Society for the Study of Evolution, 18(4), 586-608.

Espinosa Rubio, L. (2007). La vida global (en la ecobio-tecno-noos-fera). En Logos: Anales del Seminario de Metafísica, 40, 55-75. 
Galvani, P. (2001). Ecoformation et cultures amérindiennes : enjeux interculturels. Education permanente, $148,85-95$.

Galvani, P. (2002). A autoformação, uma perspectiva transpessoal, transdisciplinar e transcultural. En A. Sommerman, M. F. de Mello y V. M. de Barros (orgs.), Educação e transdisciplinaridade II (pp. 93-122). São Paulo: Triom.

Gardner, H. (1983). Frames of mind: The theory of multiple intelligence. Nueva York: Basic Books.

Goleman, D. (1995). Inteligência emocional. Rio de Janeiro: Objetiva.

Holmgren, D. (2010). Permaculture: Principles \& pathways beyond sustainability. Londres: Permanent Publications.

Janzen, D. H. (1980). When is it coevolution. Evolution, 34(3), 611-612.

Krishnamurti, J. (1966). A mutação interior. São Paulo: Cultrix.

Margulis, L. (2002). Planeta simbiótico: un nuevo punto de vista sobre la evolución. Madrid: Debate.

Maturana, H. y Varela, F. (2001). A árvore do conhecimento: as bases biológicas do conhecimento humano. São Paulo: Palas Athena.

Montessori, M. (2004). Para educar o potencial humano. São Paulo: Papirus Editora.

Morin, E. (1983). El método II: la vida de la vida. Madrid: Cátedra.

Morin, E. (1999). Los siete saberes necesarios para la educación del futuro. París: Unesco.

Naciones Unidas (2007). Declaración de las Naciones Unidas sobre los Derechos de los Pueblos Indígenas. Nueva York: Unesco.

Nicolescu, B. (2014). From modernity to cosmodernity; Science, culture, and spirituality. Nueva York: SUNY.

Norgaard, R. (1994). Development betrayed: The end of progress and a coevolutionary revisioning of the future. Nueva York: Routledge.

Novo Villaverde, M. (2009). El desarrollo sostenible: su dimensión ambiental y educativa. Madrid: Universitas.

Núñez Madrazo, M. C. (2012). Sustainability and spirituality: A transdisciplinary perspective. En B. Nicolescu (ed.), Transdisciplinarity and sustainability (pp. 102-111). Lubbock, Texas: TheATLAS Publishing.

Paul, P. (2009). Formação do sujeito e transdisciplinaridade: história de vida profissional e imaginal. São Paulo: TRIOM.

Pineau, G. (2004). Temporalidades na formação. São Paulo: Triom.

Pineau, G. (2005). Habiter la terre: ecoformation terrestre pour une conscience planétaire. París: L'Harmattan.

Riechmann, J. (2014). Un buen encaje en los ecosistemas (2. ${ }^{\mathrm{a}}$ ed. rev. de Biomímesis). Madrid: Catarata. 
ISSN $0123-1294$ | e-ISSN 2027-5358 | Educ.Educ. Vol. 20. No. 2 | Mayo-agosto de 2017 | pp. 229-248.

Universidad de La Sabana | Facultad de Educación

Robertson, R. (1992). Globalization: Social theory and global culture. Londres: SAGE.

Sahtouris, E. (1998). A Dança da Terra: Sistemas vivos em evolução: uma nova visão da biología. Rio de Janeiro: Rosa dos Tempos.

Santos, B. S. (2010). Para além do pensamento abyssal: das linhas globais a uma ecologia de saberes. En B. S. Santos y M. P. Meneses (org.), Epistemologias do Sul (pp. 31-83). São Paulo: Cortez.

Sommerman, A. (2012). A interdisciplinaridade e a transdisciplinaridade como novas formas de conhecimento para a articulação de saberes no contexto da ciência e do conhecimento em geral: contribuição para os campos da educação, da saúde e do meio ambiente (Tesis doctoral, Universidade Federal da Bahia, Salvador, Brasil).

Spier, F. (2011). El lugar del hombre en el cosmos: la gran historia y el futuro de la Humanidad. Barcelona: Crítica.

Unesco (2002). Manual de la Conferencia General. Edición de 2002 que contiene los textos y modificaciones aprobados por la Conferencia General en su 31ª reunión. París: Unesco.

Wackernagel, M.y Rees, W. (1996). Our ecological footprint: reducing human impact on the earth. Gabriola Island: New Society Publishers.

Zinkernagel, R. (2007). On observing and analyzing disease versus signals. Nature Immunology, 8(1), 8-10.

Zohar, D. (2012). Spiritual intelligence: The ultimate intelligence. Londres: Bloomsbury Publishing.

Zohar, D.y Marshall, I. (2004). Spiritual capital: Wealth we can live by. San Francisco: Berrett-Koehler Publishers. 